\title{
PTU-107 $\star$ LNA-MODIFIED OLIGONUCLEOTIDES TARGETING MRNA OF DICER IN PEGYLATED COLLOIDAL NANOPARTICLES WITH LINKED ABS AGAINST CD44 ERADICATE ESOPHAGEAL CANCER STEM CELLS (ECSC) BY INDUCTION OF PCD AFTER INHIBITION OF ONCOMIR MACHINERY
}

doi:10.1136/gut.2011.239301.235

J Giannios* Translational Cancer Medicine, Erasinio Oncology Hospital, Athens, Greece

Introduction Metastatic oesophageal squamous cell $\mathrm{Ca}$ (ESCC) is incurable due to chemoresistance caused by cancer stem cells due to overexpression of oncomirs which upregulate oncogenes and hypermethylation in $\mathrm{CpG}$ islands which inactivates tumour suppressor genes.

Methods We obtained metastatic ESCC and CSCs from patients and we injected them in xenograft animal models which were treated with LNA oligonucleotides targeting DICER where the 2'-oxygen is bridged to the 4' position via a methylene linker leading to formation of a rigid bicycle locked into a C3 endo (RNA) sugar conformation encapsulated in PEG colloidal nanoparticles with linked Abs targeting CD44. Microarray, RT-PCR, IHC, flow cytometry, MTT, BrdU, TUNEL and TEM were used.

Results There was inhibition of Dicer RNAIII endonuclease which blocked exportin5 cleavage blocking formation of mature oncogenic miRNA segments. This inhibition of oncomirs led to silencing of oncogenes such as transcription factors, apoptotic inhibitors, chromatin modifiers, growth factors (tyrosine kinases-integral membrane proteins), signal transducers (cytoplasmic regulators, membrane associated G-proteins, GTPase exchange factors and serine/threonine kinases). Dicer silencing led to inhibition of angiogenesis, invasion, metastasis, ESCC and CSC proliferation by inhibiting stem cell pathways Bmi-1, Notch, SHH and Wnt. There was inhibition of hypermethylation of $\mathrm{CpG}$ islands reactivating apoptotic tumour suppressor genes inducing irreversible D2 stage of type I PCD/apoptosis which led to a bystander killing effect. BrdU and MTT exhibited inhibition of DNA synthesis and metabolic activity of ESCC and CSCs.

Conclusion Silencing of DICER exerted a synergistic apoptotic effect by activation of tumour suppressor genes after demethylation and inhibition of oncomirs and linked oncogenes leading to eradication of chemoresistant CSCs and ESCCs.

Competing interests None.

Keywords oesophageal Ca, oncomirs, hypermethylation, stem cell pathways, Bim-1, Notch, SHH, Wnt, apoptosis. 